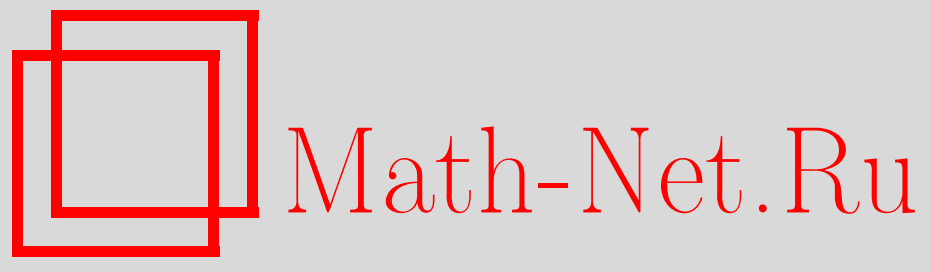

А. А. Сорокин, М-оценивание и проверка линейных гипотез в АRСН-модели, УМH, 2006, том 61, выпуск 2, 169-170

DOI: https://doi.org/10.4213/rm1735

Использование Общероссийского математического портала Math-Net.Ru подразумевает, что вы прочитали и согласны с пользовательским соглашением http://www . mathnet.ru/rus/agreement

Параметры загрузки:

IP: 34.227 .88 .159

26 апреля 2023 г., 13:14:47

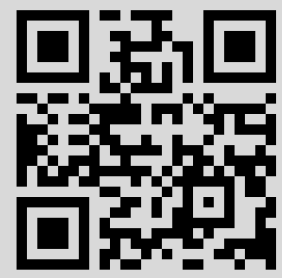




\section{M-оценивание и проверка линейных гипотез в ARCH-модели}

\section{А. А. Сорокин}

Введение. ARCH-модель является одной из наиболее популярных для исследования финансовых временных рядов. Она была предложена Р. Ф. Энглом в 1982 г. в [1] и определяется уравнениями

$$
y_{t}=\sigma_{t}(\mathbf{a}) \varepsilon_{t}, \quad \sigma_{t}^{2}(\mathbf{a})=1+a_{1} y_{t-1}^{2}+\cdots+a_{p} y_{t-p}^{2}, \quad t \in \mathbb{Z},
$$

где $\left\{y_{t}\right\}$ - наблюдения, $\mathbf{a}:=\left(a_{1}, \ldots, a_{p}\right)^{*}$ - вектор неизвестных параметров с $a_{j} \geqslant 0$ (* - знак транспонирования), $\sigma_{t}(\mathbf{a})-$ функция волатильности, $\left\{\varepsilon_{t}\right\}-$ независимые одинаково распределенные случайные величины с неизвестной функцией распределения $G(x)$.

В данной заметке мы построим и исследуем непараметрические М-оценки вектоpa a. Родственные им GM-оценки были предложены и изучены для $\mathrm{ARCH}(1)$-модели в [2]. Кроме того, мы предложим два теста для проверки гипотез о размерности уравнения (1). Один из них будет основан непосредственно на М-оценке, а второй - на функции $\mathbf{l}_{n}(\theta)$, определяющей оценку. Мы определим их мощность при локальных альтернативах.

Следующее условие является достаточным для существования стационарного решения уравнения (1), которое мы и будем рассматривать в дальнейшем:

УСловие 1. $\mathrm{E} \varepsilon_{1}=0, \mathrm{E} \varepsilon_{1}^{2}<\infty, \mathrm{E} \varepsilon_{1}^{2}\left(a_{1}+\cdots+a_{p}\right)<1$.

Построение и свойства М-оценок. Будем предполагать, что $G(x)$ абсолютно непрерывна относительно меры Лебега с плотностью $g(x)$. Положим для $t=1, \ldots, n$

$$
\mathbf{Y}_{t-1}:=\left(y_{t-1}, \ldots, y_{t-p}\right)^{*}, \quad \sigma_{t}^{2}(\theta):=1+\theta_{1} y_{t-1}^{2}+\cdots+\theta_{p} y_{t-p}^{2}, \quad \theta \in \mathbb{R}^{p} .
$$

Определим остатки в модели (1) соотношением $\varepsilon_{t}(\theta)=y_{t} / \sigma_{t}(\theta)$, если $\sigma_{t}^{2}(\theta)>0$, и положим $\varepsilon_{t}(\theta)=1$ в противном случае. Перейдем к построению М-оценок. Сначала напомним, что уравнение максимального правдоподобия (для модели (1) с начальным условием $\left.\mathbf{Y}_{0} \equiv \mathbf{0}\right)$ имеет вид

$$
n^{-1 / 2} \sum \mathbf{e}\left(\mathbf{Y}_{t-1}, \theta\right) \psi_{M L}\left(\varepsilon_{t}(\theta)\right)=\mathbf{0}
$$

где $\psi_{M L}:=1+x g^{\prime}(x) / g(x), \mathbf{e}(\mathbf{V}, \theta):=\left(e_{1}(\mathbf{V}, \theta), \ldots, e_{p}(\mathbf{V}, \theta)\right)^{*}, e_{j}(\mathbf{V}, \theta):=V_{j} /(1+$ $\left.\theta_{1} V_{1}^{2}+\cdots+\theta_{p} V_{p}^{2}\right), \mathbf{V}, \theta \in \mathbb{R}^{p}, j=1, \ldots, p$. (Здесь и ниже суммирование проводится по $t$ от 1 до $n$ и пределы берутся при $n \rightarrow \infty$.) Зафиксируем некоторую $\psi: \mathbb{R} \rightarrow \mathbb{R}$. Кажется естественным определить М-оценку как решение уравнения (2) с $\psi$ вместо неизвестной $\psi_{M L}$. Оказывается, для состоятельности такой оценки необходимо выполнение соотношений Е $\psi\left(\varepsilon_{1}\right)=0$ и $I(\psi):=\int x g(x) d \psi(x) \neq 0$. При неизвестной $g(x)$ подобрать подходящую $\psi$ не удается (см. подробности в [2]). Поэтому мы определим М-оценку при помощи уравнения

$$
\mathbf{l}_{n}(\theta):=n^{-1 / 2} \sum \mathbf{e}\left(\mathbf{Y}_{t-1}, \theta\right)\left[\psi\left(\varepsilon_{t}(\theta)\right)-\bar{\psi}_{n}(\theta)\right]=\mathbf{0},
$$

где $\bar{\psi}_{n}(\theta):=n^{-1} \sum \psi\left(\varepsilon_{t}(\theta)\right)$, избавляясь тем самым от условия $\mathrm{E} \psi\left(\varepsilon_{1}\right)=0$. Пусть $S$ - ковариационная матрица вектора $\mathbf{e}\left(\mathbf{Y}_{0}, \mathbf{a}\right), \Lambda:=-2^{-1} I(\psi) S$. Нам понадобится следующее

УСловие 2. $g(x)>0, \lim _{|x| \rightarrow \infty} x g(x)=0, \sup _{x}\left(1+x^{2}\right)\left|g^{\prime}(x)\right|<\infty, \int\left|g^{\prime}(x) x\right| d x<\infty$.

Следующая теорема устанавливает асимптотическое линейное разложение процесса $\mathbf{l}_{n}(\theta)$. Она является следствием из результатов [2] для взвешенных эмпирических процессов.

ТЕОрема 1. Пустъ выполнены условия 1 и 2 , $\mathrm{E}\left|\varepsilon_{1}\right|^{8+\delta}<\infty$ для некоторого $\delta>0$ $\left.u \operatorname{var}\right|_{-\infty} ^{\infty} \psi<\infty$. Тогда для любого $b<\infty$

$$
\sup _{\|\theta-\mathbf{a}\| \leqslant n^{-1 / 2} b}\left\|\mathbf{l}_{n}(\theta)-\mathbf{l}_{n}(\mathbf{a})-n^{1 / 2} \Lambda(\theta-\mathbf{a})\right\|=o_{p}(1) .
$$


Отметим, что утверждение теоремы 1 справедливо и для разрывных $\psi$. Прямым ее следствием является следующий результат.

Теорема 2. Пусть выполнены условия теоремы $1, \psi$ непрерывна и $I(\psi) \neq 0$. Тогда с вероятностъю, стремящейся $\kappa 1$, существует решение уравнения (3) $\widehat{\mathbf{a}}_{n}$, для которого $n^{1 / 2}\left(\widehat{\mathbf{a}}_{n}-\mathbf{a}\right)=\Lambda^{-1} \mathbf{l}_{n}(\mathbf{a})+o_{p}(1), n^{1 / 2}\left(\widehat{\mathbf{a}}_{n}-\mathbf{a}\right) \stackrel{d}{\rightarrow} \mathscr{N}(0, \Sigma)$, где $\Sigma=h^{2}(\psi) S^{-1}$, $h^{2}(\psi):=4 \mathrm{D} \psi\left(\varepsilon_{1}\right) /[I(\psi)]^{2}$.

Несложно проверить, что $\Sigma \geqslant \Sigma_{\text {opt }}$, где $\Sigma_{\text {opt }}$ соответствует случаю $\psi=\psi_{\text {opt }}:=$ $\psi_{M L}$. Следовательно, М-оценка с $\psi=\psi_{\text {opt }}$ будет иметь наименьшую матрицу ковариаций.

Проверка гипотезы о размерности модели. Пусть фиксировано некоторое $1 \leqslant q<p$. Для произвольного вектора $\theta \in \mathbb{R}^{p}$ будем полагать $\theta=\left(\theta^{1 *}, \theta^{2 *}\right)^{*}$, где $\theta^{1} \in \mathbb{R}^{q}, \theta^{2} \in \mathbb{R}^{p-q}$. Тогда гипотеза $H_{0}:=\left\{\mathbf{a}^{2}=\mathbf{0}\right\}$ состоит в том, что процесс $\left\{y_{t}\right\}$ удовлетворяет уравнению (1) размерности $q<p$. Чтобы описать альтернативу к $H_{0}$, предположим, что при $n \geqslant 1$ наблюдения удовлетворяют (1) с параметром $\mathbf{a}_{n}^{\prime}:=$ $\mathbf{a}+n^{-1 / 2} \mathbf{d}$, замещающим $\mathbf{a}$, где $\mathbf{d} \in \mathbb{R}^{p}, d_{j} \geqslant 0$. Тогда локальная альтернатива $H_{1 n}$ состоит в том, что $\mathbf{a}^{2}=\mathbf{0}, \mathbf{d}^{2} \neq \mathbf{0}$. Обычный подход для нахождения распределения тестовых статистик при локальных альтернативах использует третью лемму Ле Кама. Мы же используем аналоги теорем 1 и 2 , справедливые при $H_{1 n}$.

Пусть $\widehat{\Sigma}_{n}$ - произвольная состоятельная оценка $\Sigma$. Обозначим $\Sigma_{2,2}$ и $\widehat{\Sigma}_{n, 2,2}$ матрицы, образованные последними $(p-q)$ строками и столбцами матриц $\Sigma$ и $\widehat{\Sigma}_{n}$. Наш первый тест для проверки $H_{0}$ основан на естественной статистике $R_{n}^{2}:=n\left(\widehat{\mathbf{a}}_{n}^{2}\right)^{*} \widehat{\Sigma}_{n, 2,2}^{-1}\left(\widehat{\mathbf{a}}_{n}^{2}\right)$. Обозначим $\chi^{2}(p-q)$ и $\chi^{2}\left(p-q, b^{2}\right)$ соответственно центральное и нецентральное с параметром $b^{2}$ распределения хи-квадрат. Имеет место следующая теорема.

Теорема 3. Пусть выполнены условия теоремы 2. Тогда при гипотезе $H_{0} \quad R_{n}^{2} \stackrel{d}{\rightarrow}$ $\chi^{2}(p-q)$, а при альтернативе $H_{1 n} \quad R_{n}^{2} \stackrel{d}{\rightarrow} \chi^{2}\left(p-q, \mathbf{d}^{2 *} \Sigma_{2,2}^{-1} \mathbf{d}^{2}\right)$.

Для построения теста второго типа мы используем подход, предложенный в [3]. Пусть $\widehat{\mathbf{b}}_{n}$ - произвольная $n^{1 / 2}$-состоятельная оценка $\mathbf{a}$. Как и ранее, представим $\widehat{\mathbf{b}}_{n}$ в виде $\left(\widehat{\mathbf{b}}_{n}^{1 *}, \widehat{\mathbf{b}}_{n}^{2 *}\right)^{*}$ и положим $\widehat{\mathbf{b}}_{n}^{0}:=\left(\widehat{\mathbf{b}}_{n}^{1 *}, \mathbf{0}^{*}\right)^{*}$. Обозначим $\mathrm{Pr}$ ортогональную проекцию на последние $(p-q)$ координат. Пусть

$$
\begin{gathered}
\Sigma=\left(\begin{array}{cc}
\Sigma_{1,1} & \Sigma_{2,1}^{*} \\
\Sigma_{2,1} & \Sigma_{2,2}
\end{array}\right), \quad C:=\Sigma_{1,1}-\Sigma_{2,1}^{*} \Sigma_{2,2}^{-1} \Sigma_{2,1}, \\
V:=\left[h^{2}(\psi) \mathrm{D} \psi\left(\varepsilon_{1}\right)\right]^{-1 / 2}\left(\begin{array}{cc}
C^{1 / 2} & \Sigma_{2,1}^{*} \Sigma_{2,2}^{-1 / 2} \\
\mathbf{0} & \Sigma_{2,2}^{1 / 2}
\end{array}\right) .
\end{gathered}
$$

Можно проверить, что $V V^{*}=\left(\mathrm{D} \psi\left(\varepsilon_{1}\right) S\right)^{-1}$. Пусть $\widehat{V}_{n}-$ состоятельная оценка $V$. Рассмотрим в качестве статистики второго теста величину $Q_{n}^{2}:=n\left\|\operatorname{Pr} \widehat{V}_{n}^{*} \mathbf{l}_{n}\left(\widehat{\mathbf{b}}_{n}^{0}\right)\right\|^{2}$, где $\|\cdot\|$ - евклидова норма.

Теорема 4. Пусть выполнены условия теоремы 1 и $I(\psi) \neq 0$. Тогда при гипотезе $H_{0} \quad Q_{n}^{2} \stackrel{d}{\rightarrow} \chi^{2}(p-q)$, а при алътернативе $H_{1 n} \quad Q_{n}^{2} \stackrel{d}{\rightarrow} \chi^{2}\left(p-q, \mathbf{d}^{2 *} \Sigma_{2,2}^{-1} \mathbf{d}^{2}\right)$.

В силу теорем 3 и 4 , статистики $R_{n}^{2}$ и $Q_{n}^{2}$ асимптотически эквивалентны, но использование последней не требует непрерывности $\psi$ и построения $\widehat{\mathbf{a}}_{n}$. Отметим также, что оба теста имеют наибольшую мощность при $\psi=\psi_{\mathrm{opt}}$.

\section{Список литературы}

[1] R. F. Engle, Econometrica, 50:4 (1982), 987-1007. [2] M. V. Boldin, Math. Methods Statist., 9:1 (2000), 65-89. [3] J. P. Kreiss, Ann. Statist., 18:3 (1990), 1470-1482.

А. А. Сорокин (А. А. Sorokin)

Московский государственный университет им. М. В. Ломоносова

E-mail: aasor@mail.ru
Представлено А. В. Булинским Принято редколлегией 01.02 .2006 